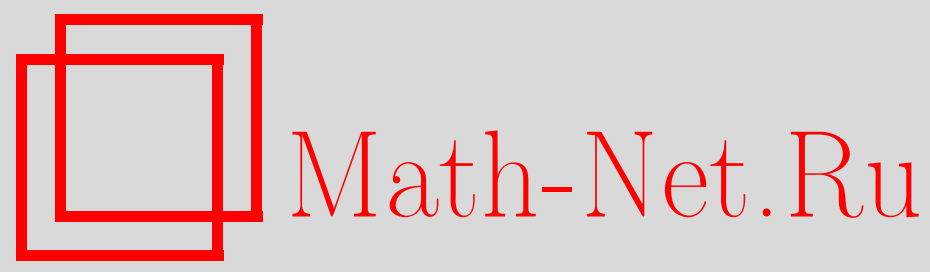

Р. О. Гринив, С. Ю. Доброхотов, А. А. Шкаликов, Операторная модель задачи о колебаниях жидкости на упругом основании, Матем. заметки, 2000, том 68, выпуск 1, 66-81

DOI: https://doi.org/10.4213/mzm920

Использование Общероссийского математического портала Math-Net.Ru подразумевает, что вы прочитали и согласны с пользовательским соглашением http://www.mathnet.ru/rus/agreement

Параметры загрузки:

IP: 54.224 .187 .69

26 апреля 2023 г., 04:12:24

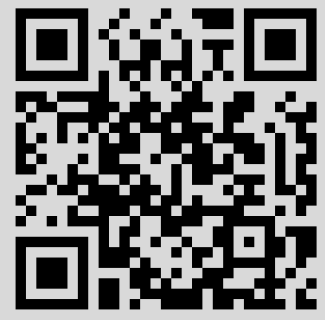




\title{
ОПЕРАТОРНАЯ МОДЕЛЬ ЗАДАЧИ О КОЛЕБАНИЯХ ЖИДКОСТИ НА УПРУГОМ ОСНОВАНИИ
}

\author{
Р. О. Гринив, С. Ю. Доброхотов, А.А. Шкаликов
}

В работе исследуется задача о малых колебаниях жидкости в слое конечной глубины на подстилающем упругом основании. Вьписаны и объяснены уравнения задачи. Основная цель работы - представить эти уравнения в форме $\mathscr{A} \ddot{w}(t)+\mathscr{T} w(t)=0$, где $\mathscr{A}$ и $\mathscr{T}$ - положительные операторы в некотором естественно связанном с задачей функциональном пространстве. Дальнейшая цель - изучить спектр линейного пучка $\lambda \mathscr{A}+\mathscr{T}$, определяющего динамику задачи.

Библиографоия: 19 названий.

Введение. Волновые движения жидкости в слое конечной глубины в большинстве известных нам работ рассматривались в предположении, что дно является абсолютно твердым. Для многих приложений это разумно. Однако есть задачи, представляющие существенный физический интерес, для которых такое предположение неприемлемо. В частности, такой является задача о поверхностных волнах, возбужденных источником, находящимся под основанием. Наиболее важным примером является задача о волнах цунами, вызываемых сейсмическими явлениями в коре земли на дне океана.

По-видимому, первым, кто начал изучение линейных моделей таких задач, был Подъяпольский [1]. Он рассмотрел весьма сложную модель, учитывающую турбулентные движения жидкости и силы гравитации внутри упругого основания, предполагая, что основание есть полупространство (плоское дно). В последующих работах [2], [3] рассматривались более упрощенные модели. Жидкость предполагалась потенциальной, а гравитационные силы в упругой среде не учитывались. Но и в такой постановке задача не была исследована достаточно полно. Здесь же отметим, что рассматриваемая задача имеет тесное отношение к теории оболочек, наполненнных жидкостью (см., например, [4]), но имеются и существенные различия.

Рассматриваемая ниже система уравнений описьвает модель, в которой жидкость предполагается идеальной и невращающейся. В отличие от [5] на поверхности жидкости учитываются силы тяжести (фактор, которьй может быть причиной возникновения поверхностных волн), и в отличие от [6] гравитационные (Архимедовы) силы учитываются также на границе раздела жидкости и упругой среды. Итак, рассматривается следующая система уравнений:

Работа выполнена при поддержке Российского фонда фундаментальных исследований, гранты № 98-01-01000, № 00-15-96100 (Р. О. Гринив и А. А. Шкаликов), грант № 99-01-00415 (С. Ю. Доброхотов). 


$$
\begin{array}{ll}
\frac{\partial^{2} v}{\partial t^{2}}+\frac{\partial v}{\partial n}=0, & x \in S_{0}, \\
\Delta v=0, & x \in \Omega_{0}, \\
\frac{\partial v}{\partial n}=u_{n}:=(\vec{u}, \vec{n}), & x \in S_{1}, \\
\rho \mu^{2} \frac{\partial^{2} v}{\partial t^{2}} \vec{n}+\mu^{2}(\rho-1) u_{3} n_{3} \vec{e}_{3}=\sigma_{n}(\vec{u}), & x \in S_{1}, \\
\mu^{2} \frac{\partial^{2} \vec{u}}{\partial t^{2}}=\Delta^{*} \vec{u}, & x \in \Omega_{1}, \\
\vec{u} \rightarrow 0 & \text { при } x_{3} \rightarrow-\infty .
\end{array}
$$

Здесь $x=\left(x_{1}, x_{2}, x_{3}\right) \in \mathbb{R}^{3} ; \Omega_{0}:=\left\{x \mid-H\left(x_{1}, x_{2}\right)<x_{3}<0\right\}$ - область, заполенная жидкостью, $S_{0}:=\left\{x \mid x_{3}=0\right\}$ - ее внешняя поверхность, $S_{1}:=\left\{x \mid x_{3}=-H\left(x_{1}, x_{2}\right)\right\}-$ поверхность раздела жидкости и упругой среды, заполняющей объем $\Omega_{1}:=\left\{x \mid x_{3}<\right.$ $\left.-H\left(x_{1}, x_{2}\right)\right\} ; H\left(x_{1}, x_{2}\right)$ - гладкая положительная функция, $\vec{n}=\left(n_{1}, n_{2}, n_{3}\right)$ - единичньй вектор нормали к $S_{0}$ и $S_{1}$, направленньй во внешнюю сторону области $\Omega_{0}$.

Функция $v=v(x, t)$ в уравнениях (0.1)-(0.5) обозначает потенциал перемещений жидкости; $\vec{u}=\vec{u}(x, t)$ - вектор смещений упругого дна; $\sigma_{n}(\vec{u})$ - вектор нормальных граничных напряжений с компонентами

$$
\sigma_{i n}(\vec{u})=\sum_{j=1}^{3} \sigma_{i j}(\vec{u}) n_{j}
$$

где

$$
\sigma_{i j}(\vec{u})=\left(c^{2}-2\right) \delta_{i j} \operatorname{div} \vec{u}+\frac{\partial u_{i}}{\partial x_{j}}+\frac{\partial u_{j}}{\partial x_{i}}, \quad i, j=1,2,3,
$$

- тензор напряжений; $\Delta^{*} \vec{u}=\Delta \vec{u}+\left(c^{2}-1\right) \nabla \operatorname{div} \vec{u}$ - дифференциальньй оператор теории упругости; $\overrightarrow{e_{3}}:=(0,0,1) ; \rho:=\rho_{w} / \rho_{c}<1$ - отношение плотности жидкости $\rho_{w}$ и плотности коры земли $\rho_{c} ; c:=c_{l} / c_{t}>\sqrt{2}$ - отношение скоростей продольных и поперечных волн в коре; $\mu:=L g / c_{t} ; L$ - характерньй размер, $g$ - ускорение свободного падения.

Преж де чем формулировать основные цели статьи, поясним, что уравнения $(0.1)-(0.5)$ могут быть получены с помощью следующего вариационного принципа (мы используем безразмерные координаты). Пусть $\vec{u}(x, t), x \in \Omega_{0},-$ вектор смещений жидкости. Если жидкость предполагается потенциальной, то (как обьчно, точка означает дифференцирование по времени)

$$
\operatorname{div} \overrightarrow{\dot{u}}=0 \quad \text { при } x \in \Omega_{0} .
$$

$\Phi$ ункция $\vec{u}(x, t)$ на поверхности основания $S_{1}$ удовлетворяет условию непротекания

$$
\left(\vec{u}^{+}, \vec{n}\right)=\left(\vec{u}^{-}, \vec{n}\right) \quad \text { при } x \in S_{1},
$$

где $\vec{u}^{+}$и $\vec{u}^{-}-$граничные значения вектора $\vec{u}(x, t)$ на $S_{1}$ сверху и снизу соответственно.

Кинетическая энергия $T$ рассматриваемой системы равна

$$
T=\frac{\rho_{w}}{2} \int_{\Omega_{0}}|\overrightarrow{\dot{u}}|^{2} d x+\frac{\rho_{c}}{2} \int_{\Omega_{1}}|\overrightarrow{\dot{u}}|^{2} d x .
$$


Потенциальная энергия состоит из энергии напряжений упругой коры, силы притяжения на поверхности жидкости и на границе раздела жидкости и упругой среды (энергии архимедовых сил, ср. [7]-[10]):

$$
\begin{aligned}
\Pi= & \frac{\rho_{c}}{2} \int_{\Omega_{1}}\left\{c_{t}^{2} \sum_{i=1}^{3}\left(\left|\nabla u_{i}\right|^{2}+\sum_{j=1}^{3} \frac{\partial u_{i}}{\partial x_{j}} \frac{\partial u_{j}}{\partial x_{i}}\right)+\left(c_{l}^{2}-2 c_{t}^{2}\right)|\operatorname{div} \vec{u}|^{2}\right\} d x \\
& +\frac{\rho_{w}}{2} \int_{S_{0}}\left|u_{3}\right|^{2} d s+\frac{\rho_{w}-\rho_{c}}{2} \int_{S_{1}} n_{3}\left|u_{3}\right|^{2} d s .
\end{aligned}
$$

Рассмотрим вариационную задачу

$$
\int_{t_{1}}^{t_{2}} L_{0}(t, u, \dot{u}, \nabla u) d t=\int_{t_{1}}^{t_{2}}(T-\Pi) d t \rightarrow \min , \quad t_{1}<t_{2},
$$

с граничньпи условиями

$$
L_{0}\left(t_{1}\right)=L_{0}\left(t_{2}\right)=0
$$

и связями $(0.7),(0.8)$. Следуя [9], [10], введем множители Лагранжа $\dot{v}(x, t)$ и $\varphi(x, t)$ в условия (0.7) и (0.8) соответственно (для удобства мы берем $\dot{v}$ вместо $v$ ). Тогда получим эквивалентную задачу с лагранжианом

$$
L(t, \vec{u}, \overrightarrow{\dot{u}}, \nabla \vec{u}, \nabla \overrightarrow{\dot{u}}, \dot{v}, \varphi)=T-\Pi+\int_{\Omega_{0}} \dot{v} \operatorname{div} \overrightarrow{\dot{u}} d x+\int_{S_{1}} \varphi\left(\vec{u}^{+}-\vec{u}^{-}, \vec{n}\right) d s .
$$

Лемма. Уравнение Эйлера-Лагранжа для вариационной задачи

$$
\int_{t_{1}}^{t_{2}} L(t) d t \rightarrow \min , \quad L\left(t_{1}\right)=L\left(t_{2}\right)=0
$$

записанной в безразмерных координатах, совпадает с системой (0.1)-(0.5). Более того, $u=\nabla v$ в $\Omega_{0} и \varphi=\rho_{w} \ddot{v} н а S_{1}$.

ДокАЗАТЕЛЬСтво. Нужно применить формулу Стокса к уравнению Эйлера-Лагранжа, исключить $\varphi$, выразить $\vec{u}(x, t)$ при $x \in \Omega_{0}$ в терминах функции $v(x, t)$ и перейти к безразмерным координатам $t=\sqrt{L / g} t_{1}, x=L x_{1}, v=L^{2} v_{1}$ и $\vec{u}=L \vec{u}_{1}$. Все вычисления проводятся непосредственно и здесь опущены.

Основная цель статьи - представить задачу (0.1)-(0.6) в операторном виде

$$
\mathscr{A} \ddot{w}(t)+\mathscr{T} w(t)=0,
$$

где $\mathscr{A}$ и $\mathscr{T}$ - самосопряженные операторы в подходящем гильбертовом пространстве $\mathscr{H}$, и изучить спектр линейного пучка $\lambda \mathscr{A}+\mathscr{T}$, который в существенном определяет динамику уравнения (0.10). Важнейший момент при реализации операторной трактовки задачи - выбор функций, выступающих в качестве элементов функционального пространства, и конструкция самих пространств. Один такой выбор - функции $v_{0}:=\left.v\right|_{S_{0}}$, $v_{1}:=\left.v\right|_{S_{1}}$ и $\vec{u}$ - был предложен в [11]. Однако при таком выборе саму задачу не удалось представить в форме (0.10) с самосопряженными коэффициентами, хотя в [11] была найдена последовательность задач с самосопряженными коэффициентами, аппроксимирующих исходную. 
Мы предлагаем другой выбор переменных, которьй представляется более естественным как с физической, так и с математической точек зрения. А именно, в качестве элементов ниже конструируемого гильбертова пространства $\mathscr{H}$ выступают вектор-функции

$$
\vec{w}(x, t)=\left(\begin{array}{c}
\eta\left(x_{1}, x_{2}, t\right) \\
\vec{u}\left(x_{1}, x_{2}, x_{3}, t\right)
\end{array}\right),
$$

где $\eta:=\left.\left(\partial v / \partial x_{3}\right)\right|_{S_{0}}$ - вертикальныеперемещения жидкости относительно невозмущенной поверхности $S_{0}$. При этом операторы $\mathscr{A}$ и $\mathscr{T}$ оказьваются положительными в $\mathscr{H}$, а энергия системы

$$
(\mathscr{A} \dot{w}, \dot{w})_{\mathscr{H}}+(\mathscr{T} w, w)_{\mathscr{H}}
$$

совпадает с $T-\Pi$ в (0.9) и оказывается независимой от времени. Это подтверждает правильность выбора переменных и функционального пространства. Отметим, что в работах [2], [9], [11] оценки решений системы по времени $t$ получались экспоненциальными.

1. Функциональные пространства. Вспомогательные результаты. Пусть $\Omega \subset \mathbb{R}^{3}$ - неограниченная область с равномерно липшицевой границей $\partial \Omega$. Напомним $[12, \S 1.1 .9]$, что это условие означает следующее: в каждой точке $x \in \partial \Omega$ найдется окрестность $U$ такая, что в некоторой декартовой системе координат $\left(y_{1}, y_{2}, y_{3}\right)$ множество $U \cap \Omega$ определяется неравенством $y_{3}<f_{x}\left(y_{1}, y_{2}\right)$, где функция $f_{x}$ удовлетворяет условию Липшица

$$
\left|f_{x}(y)-f_{x}\left(y^{\prime}\right)\right| \leqslant C\left|y-y^{\prime}\right|
$$

причем постоянная $C$ не зависит от $x$.

Через $W_{2}^{1}(\Omega)$ обозначим пространство Соболева с нормой

$$
\|u\|_{W_{2}^{1}(\Omega)}=\left(\int_{\Omega}\left(|\nabla u|^{2}+|u|^{2}\right) d x\right)^{1 / 2} .
$$

Обозначение $L_{2}^{1}(\Omega)$ резервируем для функций $u(x)$ таких, что $\partial u / \partial x_{j} \in L_{2}(\Omega)$ для всех $j=1,2,3$. Через $V_{2}^{1}(\Omega)$ обозначим пополнение пространства $W_{2}^{1}(\Omega)$ по норме

$$
\|u\|_{V_{2}^{1}(\Omega)}=\left(\int_{\Omega}|\nabla u|^{2} d x\right)^{1 / 2}
$$

(так как $\Omega$ - бесконечная область, то $W_{2}^{1}(\Omega)$ не содержит ненулевых констант, поэтому $(1.2)$ - норма). Очевидно, $V_{2}^{1}(\Omega)$ содержит $W_{2}^{1}(\Omega)$, но не содержится в $L_{2}(\Omega)$. Например, если $\Omega=\mathbb{R}^{3}$ и $\chi(x)$ - гладкая функция, аннулирующаяся в окрестности начала координат, то $\chi(x)|x|^{-1-\varepsilon} \in V_{2}^{1}(\Omega)$ не принадлежит $L_{2}(\Omega)$. Однако согласно неравенству Соболева

$$
\|u\|_{L_{p}(\Omega)} \leqslant C\|\nabla u\|_{L_{2}(\Omega)}, \quad p \geqslant 6
$$

поэтому $V_{2}^{1}(\Omega)$ есть подмножество $L_{6}(\Omega)$.

ЗАмЕчАниЕ 1.1. Неравенство (1.3) доказано в [12] для бесконечно гладких функций с компактным носителем в $\Omega$. Чтобы показать, что оно остается справедливым для всех функций из $V_{2}^{1}(\Omega)$, воспользуемся следующим результатом $[13$, гл. VI, §3]. Для областей $\Omega$ с равномерно липшицевой границей существует ограниченный оператор продолжения

$$
\mathscr{E}: V_{2}^{1}(\Omega) \cap L_{6}(\Omega) \rightarrow V_{2}^{1}\left(\mathbb{R}^{3}\right) \cap L_{6}\left(\mathbb{R}^{3}\right)
$$

Поэтому для $u \in V_{2}^{1}(\Omega)$ и $\widetilde{u}:=\mathscr{E} u \in V_{2}^{1}\left(\mathbb{R}^{3}\right)$ справдлива оценка

$$
\|u\|_{L_{6}(\Omega)} \leqslant\|\widetilde{u}\|_{L_{6}\left(\mathbb{R}^{3}\right)} \leqslant C\|\widetilde{u}\|_{V_{2}^{1}\left(\mathbb{R}^{3}\right)} \leqslant C_{1}\|u\|_{V_{2}^{1}(\Omega)} .
$$

Полезно отметить следуюший факт. 
ПРЕДЛОЖЕНИЕ 1.1. Имеем $V_{2}^{1}(\Omega)=L_{6}(\Omega) \cap L_{2}^{1}(\Omega)$.

ДокАЗАТЕЛЬСтво. Включение $V_{2}^{1}(\Omega) \subset L_{6}(\Omega) \cap L_{2}^{1}(\Omega)$ следует из (1.2) и (1.3). Обратно, пусть $u \in L_{6}(\Omega) \cap L_{2}^{1}(\Omega)$. Выберем функции $\eta(x)$ и $\nu(x) \in C_{0}^{\infty}\left(\mathbb{R}^{3}\right)$ такие, что $\eta(x) \geqslant 0, \int \eta(x) d x=1,0 \leqslant \nu(x) \leqslant 1, \nu(x)=1$ при $|x| \leqslant 1$, и положим $\eta_{\delta}(x):=\delta^{-3} \eta(x / \delta), \nu_{\varepsilon}(x):=\nu(\varepsilon x)$. Тогда семейство функций

$$
u_{\varepsilon, \delta}(x):=\nu_{\varepsilon}(x) u(x) * \eta_{\delta}(x)
$$

(здесь “*” - свертка функций) обладает свойствами

$$
u_{\varepsilon, \delta}(x) \in C_{0}^{\infty}\left(\mathbb{R}^{3}\right), \quad \lim _{\varepsilon \rightarrow 0} \lim _{\delta \rightarrow 0} \nabla u_{\varepsilon, \delta}(x)=\nabla u(x) \quad \text { в }\left(L_{2}\left(\mathbb{R}^{3}\right)\right)^{3} .
$$

Первое утверждение очевидно. Для доказательства второго утверждения заметим, что $\nabla u_{\varepsilon, \delta}(x) \rightarrow \nabla\left(\nu_{\varepsilon} u(x)\right)$ при $\delta \rightarrow 0$ в норме пространства $\left(L_{2}\left(\mathbb{R}^{3}\right)\right)^{3}$ (см. [13, гл. III, $\left.\left.\S 2\right]\right)$. Далее,

$$
\nabla\left(\nu_{\varepsilon} u(x)\right)=\nabla \nu_{\varepsilon} \cdot u(x)+\nu_{\varepsilon} \cdot \nabla u(x) \rightarrow \nabla u(x) \quad \text { при } \varepsilon \rightarrow 0
$$

в норме $\left(L_{2}\left(\mathbb{R}^{3}\right)\right)^{3}$, поскольку $\nu_{\varepsilon} \rightarrow 1$ и

$$
\begin{aligned}
& \left\|\nabla \nu_{\varepsilon} \cdot u(x)\right\|_{L_{2}\left(\mathbb{R}^{3}\right)}=\int_{\mathbb{R}^{3}}\left|\nabla \nu_{\varepsilon}(x)\right|^{2}|u(x)|^{2} d x \\
& \quad \leqslant\left(\int_{|\varepsilon x| \geqslant 1}\left|\nabla \nu_{\varepsilon}(x)\right|^{3} d x\right)^{2 / 3}\left(\int_{|\varepsilon x| \geqslant 1}|u(x)|^{6} d x\right)^{1 / 3} \rightarrow 0 \quad \text { при } \varepsilon \rightarrow 0 .
\end{aligned}
$$

Последнее неравенство следует из условия $u(x) \in L_{6}(\Omega)$ и неравенства

$$
\int_{|\varepsilon x| \geqslant 1}\left|\nabla \nu_{\varepsilon}(x)\right|^{3} d x \leqslant \int_{\mathbb{R}^{3}}\left|\nabla_{y} \nu(y)\right|^{3} d y<\infty .
$$

Итак, ограничения функций $u_{\varepsilon, \delta}(x)$ на $\Omega$ сходятся к $u(x)$ при $\varepsilon, \delta \rightarrow 0$ в $V_{2}^{1}(\Omega)$-норме, а потому $u(x) \in V_{2}^{1}(\Omega)$.

ЗАМЕЧАнИЕ 1.2. Аналогично вводятся пространства $V_{2}^{s}(G)$ при любом $s \geqslant 0$ и области $G \subset \mathbb{R}^{n}$ с равномерно липшищевой границей $\partial G$. Пространство $V_{2}^{s}\left(\mathbb{R}^{n}\right)$ есть пополнение $C_{0}^{\infty}\left(\mathbb{R}^{n}\right)$ по норме

$$
\|u\|_{V_{2}^{s}\left(\mathbb{R}^{n}\right)}=\left\|\frac{1}{(2 \pi)^{n}} \iint|p|^{s} e^{i p(x-y)} u(y) d y d p\right\|_{L_{2}\left(\mathbb{R}^{n}\right)},
$$

где $p=\left(p_{1}, \ldots, p_{n}\right) \in \mathbb{R}^{n}$ и $|p|=\sqrt{p_{1}^{2}+\cdots+p_{n}^{2}}$. Пространство $V_{2}^{s}(G), G \subset \mathbb{R}^{n}$, можно определить как совокупность сужений на область $G$ функций из $V_{2}^{s}\left(\mathbb{R}^{n}\right)$ с нормой факторпространства. Если $G$ - неограниченная область и $s<n / 2$, то $V_{2}^{s}(G)$ - гильбертово пространство и совпадает с $L_{q}(G) \cap L_{2}^{s}(G)$, где $q=2 n /(n-2 s)$. Доказательство проводится так же, как в предложении 1.1 ; обобщение неравенства (1.3) имеется в $[12$, $\S 1.4 .1]$.

Далее, для области $G$ с равномерно липшицевой границей оператор взятия следа $\Gamma: C^{\infty}(\bar{G}) \rightarrow C(\partial G)$ продолжается при $s>1 / 2$ как ограниченньй оператор (см. [13, гл. VI])

$$
\Gamma: V_{2}^{s}(G) \rightarrow V_{2}^{s-1 / 2}(\partial G) .
$$

Этот оператор сюрьективен, т.е. существует оператор продолжения $e: V_{2}^{s-1 / 2}(\partial G) \rightarrow$ $V_{2}^{s}(G)$ такой, что Г о е есть единичньй оператор в $V_{2}^{s-1 / 2}(\partial G)$.

Докажем еше одну важную лемму. Для функций $\vec{u} \in \mathbb{W}_{2}^{1}(\Omega):=\left(W_{2}^{1}(\Omega)\right)^{3}$ положим

$$
e_{i j}(\vec{u}):=\frac{\partial u_{i}}{\partial x_{j}}+\frac{\partial u_{j}}{\partial x_{i}}, \quad e(\vec{u}):=\left(\sum_{i, j=1}^{3}\left|e_{i j}(\vec{u})\right|^{2}\right)^{1 / 2}
$$


ПРЕДЛОЖЕНИЕ 1.2. Пусть $\Omega$ - неограниченная область с равномерно липиииевой граничей $\partial \Omega$. Тогда норма

$$
\|\vec{u}\|_{E(\Omega)}:=\left(\int_{\Omega}|e(\vec{u})|^{2} d x\right)^{1 / 2}
$$

әквивалентна норме пространства $\mathbb{V}_{2}^{1}(\Omega):=\left(V_{2}^{1}(\Omega)\right)^{3}$. Более того, справедливо неравенство

$$
2\|\vec{u}\|_{\mathbb{V}_{2}^{1}(\Omega)}^{2} \leqslant\|\vec{u}\|_{E(\Omega)}^{2} \leqslant 4\|\vec{u}\|_{\mathbb{V}_{2}^{1}(\Omega)}^{2}
$$

ДокАЗАТЕЛьСТво. Правая оценка в (1.4) следует из неравенства

$$
\left|e_{i j}(\vec{u})\right|^{2} \leqslant 2\left|\frac{\partial u_{i}}{\partial x_{j}}\right|^{2}+2\left|\frac{\partial u_{j}}{\partial x_{i}}\right|^{2}
$$

Левая оценка - неравенство типа Корна. Заметим, что множество $\left(C_{0}^{\infty}(\bar{\Omega})\right)^{3}$ гладких функций с компактньм носителем в $\bar{\Omega}$ является плотным подмножеством в $\mathbb{W}_{2}^{1}(\Omega)$ в силу существования ограниченного оператора продолжения $\mathscr{E}: W_{2}^{1}(\Omega) \rightarrow W_{2}^{1}\left(\mathbb{R}^{3}\right)$ (cp. [13, гл. VI, § 3]). Поэтому достаточно доказать $(1.4)$ для $\vec{u} \in\left(C_{0}^{\infty}(\bar{\Omega})\right)^{3}$. Пусть

$$
\vec{\eta}=\left(\eta_{1}, \eta_{2}, \eta_{3}\right) \in\left(C_{0}^{\infty}(\bar{\Omega})\right)^{3}, \quad \eta_{j}(x) \geqslant 0, \quad \int \eta_{j}(x)=1, \quad \vec{\eta}_{\varepsilon}(x):=\varepsilon^{-3} \vec{\eta}\left(\frac{x}{\varepsilon}\right)
$$

Положим $\vec{u}_{\varepsilon}(x):=\vec{u}(x) * \vec{\eta}_{\varepsilon}(x) \in\left(C_{0}^{\infty}\left(\mathbb{R}^{3}\right)\right)^{3}$. Тогда для $\vec{u}_{\varepsilon}(x)$ неравенство $(1.4)$ вьполняется согласно [14]. Так как $\vec{u}_{\varepsilon}(x) \rightarrow \vec{u}(x)$ в $\mathbb{W}_{2}^{1}(\Omega)$ при $\varepsilon \rightarrow 0$ (ср. [13]), то (1.4) остается справедливьм для всех функций $\vec{u}(x) \in \mathbb{V}_{2}^{1}(\Omega)$.

В следующих двух пунктах мы построим операторы, которые играют основную роль в редукции задачи (0.1)-(0.5) к операторной модели. Оператор $\mathbb{A}$ связан с первьми тремя уравениями задачи и определен в пространстве $L_{2}\left(S_{0}\right) \times L_{2}\left(S_{1}\right)$, а операторы $\mathbb{D}, \mathbb{B}_{0}$ и $\mathbb{B}_{1}$ порождены уравнениями в упругом основании и на его границе.

2. Конструкция оператора $\mathbb{A}$. Пусть $\stackrel{\circ}{V}_{2}^{1}\left(\Omega_{0}\right)$ - замькание $C_{0}^{\infty}\left(\Omega_{0}\right)$ в норме $(1.2)$. Поскольку область $\Omega_{0}$ ограничена в направлении $x_{3}$-оси, для функций из $C_{0}^{\infty}\left(\Omega_{0}\right)$ выполняется неравенство Фридрихса. Следовательно, $\stackrel{\circ}{2}_{2}^{1}\left(\Omega_{0}\right)$ совпадает с пространством $\stackrel{\circ}{2}_{2}^{1}\left(\Omega_{0}\right)$, являющимся пополнением $C_{0}^{\infty}\left(\Omega_{0}\right)$ по норме $(1.1)$, и ненулевым является подпространство $\widetilde{V}_{2}^{1}\left(\Omega_{0}\right):=V_{2}^{1}\left(\Omega_{0}\right) \ominus \stackrel{\circ}{V}_{2}^{1}\left(\Omega_{0}\right)$, где ортогональность понимается в (1.2)-норме. Поскольку $\stackrel{\circ}{2}_{2}^{1}\left(\Omega_{0}\right)$ - ядро оператора следа $\Gamma$, то $\Gamma$ отображает $\widetilde{V}_{2}^{1}\left(\Omega_{0}\right)$ на $V_{2}^{1 / 2}\left(\partial \Omega_{0}\right)$ биективно. Обозначим через $V_{2}^{-1 / 2}\left(\partial \Omega_{0}\right)$ пространство, дуальное к $V_{2}^{1 / 2}\left(\partial \Omega_{0}\right)$ по отношению к $L_{2}\left(\partial \Omega_{0}\right)$, а через $\Gamma_{1}$ замыкание в $V_{2}^{1}\left(\Omega_{0}\right)$ оператора следа нормальной производной $\left.u \rightarrow(\partial u / \partial n)\right|_{\partial \Omega_{0}}$. Покажем, что оператор

$$
\Gamma_{1}: \widetilde{V}_{2}^{1}\left(\Omega_{0}\right) \rightarrow V_{2}^{-1 / 2}\left(\partial \Omega_{0}\right)
$$

есть биекция. Сначала напомним известные понятия. 
ОПРЕДЕЛЕНИЕ 2.1. Функция $v \in V_{2}^{1}\left(\Omega_{0}\right)$ называется обобщенным решением задачи

$$
\begin{array}{ll}
\Delta v=0 & \text { в } \Omega_{0}, \\
\Gamma_{1} v=f & \text { на } \partial \Omega_{0},
\end{array}
$$

если для любого $w \in V_{2}^{1}\left(\Omega_{0}\right)$ выполняется равенство

$$
\int_{\Omega_{0}} \nabla w \nabla \bar{v} d x=\int_{\partial \Omega_{0}}(\Gamma w) \bar{f} d s
$$

Конечно, если обобщенное решение $v$ - гладкая функция, то в силу формулы Грина она является классическим решением задачи (2.1), (2.2). Заметим, что в силу (2.3) любое обобщенное решение ортогонально пространству $\stackrel{\circ}{2}_{2}^{1}\left(\Omega_{0}\right)$ и потому принадлежит $\widetilde{V}_{2}^{1}\left(\Omega_{0}\right)$.

Лемма 2.1. Для любой функиии $f \in V_{2}^{-1 / 2}\left(\partial \Omega_{0}\right)$ существует единственное обобщенное решение задачи (2.1), (2.2).

ДокАЗАТЕЛЬСТво. Если $f \in V_{2}^{-1 / 2}\left(\partial \Omega_{0}\right)$, то

$$
\left|\int_{\partial \Omega_{0}}(\Gamma w) \bar{f} d s\right| \leqslant\|\Gamma w\|_{V_{2}^{1 / 2}\left(\partial \Omega_{0}\right)}\|f\|_{V_{2}^{-1 / 2}\left(\partial \Omega_{0}\right)} \leqslant C\|w\|_{\tilde{V}_{2}^{1}\left(\Omega_{0}\right)}\|f\|_{V_{2}^{-1 / 2}\left(\partial \Omega_{0}\right)},
$$

а потому правая часть (2.3) определяет линейньй ограниченньй функционал в пространстве $\widetilde{V}_{2}^{1}\left(\Omega_{0}\right)$. Согласно теореме Рисса обобщенное решение существует и единственно.

СлЕДСТВИЕ 2.1. Оператор $\Gamma_{1}$ осуществляет гомеоморфизм пространств $\widetilde{V}_{2}^{1}\left(\Omega_{0}\right) u V_{2}^{-1 / 2}\left(\partial \Omega_{0}\right)$.

ДокАЗАТЕЛЬСтво. Согласно лемме $2.1 \Gamma_{1}$ есть биекция указанных пространств. Кроме того, в силу (2.4) выполняется оценка

$$
\|v\|_{\tilde{V}_{2}^{1}\left(\Omega_{0}\right)} \leqslant C\left\|\Gamma_{1} v\right\|_{V_{2}^{-1 / 2}\left(\partial \Omega_{0}\right)}
$$

т.е. $\Gamma_{1}$ - ограниченньй оператор. В силу теоремы Банаха он ограниченно обратим.

Определим теперь оператор $\mathbb{A}$ равенством

$$
\mathbb{A}:=\Gamma \circ \Gamma_{1}^{-1}: V_{2}^{-1 / 2}\left(\partial \Omega_{0}\right) \rightarrow V_{2}^{1 / 2}\left(\partial \Omega_{0}\right) .
$$

Другими словами, $\mathbb{A} f=\Gamma v$, где $v$ - обобщенное решение задачи $(2.1),(2.2)$. В силу равенства

$$
\int_{\partial \Omega_{0}}(\mathbb{f} f) \bar{f} d s=\int_{\Omega_{0}}|\nabla v|^{2} d x>0
$$

оператор $\mathbb{A}$ симметричен и положителен в пространстве $L_{2}(S)=L_{2}\left(S_{0}\right) \times L_{2}\left(S_{1}\right)$. В этом произведении пространств он имеет естественное матричное представление

$$
\mathbb{A}=\left(\begin{array}{cc}
A_{00} & A_{01} \\
A_{10} & A_{11}
\end{array}\right)
$$

где $A_{i j} \in \mathscr{L}\left(V_{2}^{-1 / 2}\left(S_{j}\right), V_{2}^{1 / 2}\left(S_{i}\right)\right)$ (здесь через $\mathscr{L}\left(\mathscr{H}_{1}, \mathscr{H}_{2}\right)$ обозначается пространство ограниченных операторов из $\mathscr{H}_{1}$ в $\left.\mathscr{H}_{2}\right)$. Например, $A_{00} f_{0}=\left.v_{0}\right|_{S_{0}}$, где $v_{0}$ - решение задачи $(2.1),(2.2)$ для $f=\left\{f_{0}, 0\right\}$. 
3. Конструкция операторов $\mathbb{D}, \mathbb{B}_{0}, \mathbb{B}_{1}$. Обозначим через $N$ оператор скалярного умножения на единичный нормальный вектор в $\left(L_{2}\left(S_{1}\right)\right)^{3}, N \vec{v}=(\vec{v}, \vec{n})$. Введем также операторы

$$
\vec{N} \psi=\vec{n} \psi, \quad \psi \in L_{2}\left(S_{1}\right), \quad \vec{N}_{3} \vec{u}=n_{3} u_{3} \vec{e}_{3}, \quad \vec{u}=\left(u_{1}, u_{2}, u_{3}\right) \in\left(L_{2}\left(S_{1}\right)\right)^{3} .
$$

$\mathrm{C}$ учетом представления (2.5) оператора $\mathbb{A}$ перепишем систему (0.1)-(0.5) в виде

$$
\begin{gathered}
\frac{\partial^{2}}{\partial t^{2}}\left(A_{00} \eta+A_{01} N \Gamma \vec{u}\right)+\eta=0, \\
\rho \mu^{2} \frac{\partial^{2}}{\partial t^{2}} \vec{N}\left(A_{10} \eta+A_{11} N \Gamma \vec{u}\right)+\mu^{2}(\rho-1) \vec{N}_{3} \Gamma \vec{u}=\sigma_{n}(\vec{u}), \\
\mu^{2} \frac{\partial^{2} \vec{u}}{\partial t^{2}}=\Delta^{*} \vec{u},
\end{gathered}
$$

где $\eta:=\left.(\partial v / \partial n)\right|_{S_{0}}, \vec{u} \in\left(V_{2}^{1}\left(\Omega_{1}\right)\right)^{3}$ и $\Gamma \vec{u}=\left.\vec{u}\right|_{S_{1}}$.

Отметим, что уравнения (3.1)-(3.3) уже можно записать в операторной форме $\mathscr{A}^{\prime} u(t)$ - $\mathscr{T}^{\prime} \ddot{u}(t)$ в пространстве $L_{2}\left(S_{0}\right) \times\left(L_{2}\left(S_{1}\right)\right)^{3} \times\left(L_{2}\left(\Omega_{1}\right)\right)^{3}$ (см. ниже п. 5). Однако, мы не можем показать симметричность и положительность операторов $\mathscr{A}^{\prime}$ и $\mathscr{T}^{\prime}$.

Продолжим работу. В пространстве $\mathrm{E}_{2}\left(\Omega_{1}\right):=\left(L_{2}\left(S_{1}\right)\right)^{3} \times\left(L_{2}\left(\Omega_{1}\right)\right)^{3}$ рассмотрим оператор $\mathbb{D}_{0}$, определенный на области

$$
\mathscr{D}\left(\mathbb{D}_{0}\right)=\left\{\left(\begin{array}{c}
\vec{u}_{\Gamma} \\
\vec{u}
\end{array}\right) \mid \vec{u} \in \mathbb{W}_{2}^{2}\left(\Omega_{1}\right), \vec{u}_{\Gamma}=\Gamma \vec{u}\right\}
$$

равенством

$$
\mathbb{D}_{0}\left(\begin{array}{c}
\vec{u}_{\Gamma} \\
\vec{u}
\end{array}\right)=\left(\begin{array}{c}
-\sigma_{n}(\vec{u}) \\
-\Delta^{*} \vec{u}
\end{array}\right) .
$$

Лемма 3.1. Оператор $\mathbb{D}_{0}$ положителен в пространстве $\mathrm{七}_{2}\left(\Omega_{1}\right)$. Более того, справедлива оченка

$$
\|\vec{u}\|_{V_{2}^{1}\left(\Omega_{1}\right)}^{2} \leqslant\left(\mathbb{D}_{0} U, U\right) \leqslant \mathrm{const}\|\vec{u}\|_{V_{2}^{1}\left(\Omega_{1}\right)}^{2}, \quad U=\left(\begin{array}{c}
\vec{u}_{\Gamma} \\
\vec{u}
\end{array}\right),
$$

əде $\|\cdot\|_{\mathbb{V}_{2}^{1}\left(\Omega_{1}\right)}$ - норма в пространстве $\mathbb{V}_{2}^{1}\left(\Omega_{1}\right):=\left(V_{2}^{1}\left(\Omega_{1}\right)\right)^{3}$.

ДокАЗАТЕЛЬСТво. Заметим, что

$$
\left(\Delta^{*} \vec{u}\right)_{j}=\sum_{k=1}^{3} \frac{\partial \sigma_{j k}(\vec{u})}{\partial x_{k}} .
$$

Интегрируя по частям, находим

$$
\begin{aligned}
-\left(\Delta^{*} \vec{u}, \vec{u}\right) & =-\sum_{j, k=1}^{3} \int_{\Omega_{1}} \frac{\partial \sigma_{j k}(\vec{u})}{\partial x_{k}} \bar{u}_{j} d x \\
& =-\sum_{j, k=1}^{3}\left(\int_{\Omega_{1}} \frac{\partial}{\partial x_{k}}\left(\sigma_{j k}(\vec{u}) \bar{u}_{j}\right) d x-\int_{\Omega_{1}} \sigma_{j k}(\vec{u}) \frac{\partial \bar{u}_{j}}{\partial x_{k}} d x\right) \\
& =\int_{S_{1}}\left(\sigma_{n}(\vec{u}), \Gamma \vec{u}\right) d s+\left(c^{2}-2\right) \int_{\Omega_{1}}|\operatorname{div} \vec{u}|^{2} d x+\frac{1}{2} \int_{\Omega_{1}} \sum_{j, k=1}^{3}\left|\frac{\partial \vec{u}_{i}}{\partial x_{k}}+\frac{\partial \vec{u}_{k}}{\partial x_{i}}\right|^{2} d x .
\end{aligned}
$$


Согласно предложению 1.2 и неравенству $c \geqslant \sqrt{2}$ в результате получаем

$$
\begin{aligned}
\left(\mathbb{D}_{0} U, U\right) & =-\left(\Delta^{*} \vec{u}, \vec{u}\right)-\left(\sigma_{n}(\vec{u}), \Gamma \vec{u}\right)_{S_{1}} \\
& =\left(c^{2}-2\right) \int_{\Omega_{1}}|\operatorname{div} \vec{u}|^{2} d x+\frac{1}{2} \int_{\Omega_{1}} \sum_{j, k=1}^{3}\left|\frac{\partial \vec{u}_{i}}{\partial x_{k}}+\frac{\partial \vec{u}_{k}}{\partial x_{i}}\right|^{2} d x \\
\geqslant\|\vec{u}\|_{\mathbb{V}_{2}^{1}\left(\Omega_{1}\right)}^{2} . &
\end{aligned}
$$

Оценка сверху формы $\left(\mathbb{D}_{0} U, U\right)$ очевидна. Лемма доказана.

Обозначим через $\mathbb{H}_{D}$ замыкание пространства $\mathbb{W}_{2}^{2}\left(\Omega_{1}\right)$ по норме

$$
\|\vec{u}\|_{D}^{2}:=\left(\mathbb{D}_{0} U, U\right), \quad U=\left(\begin{array}{c}
\vec{u}_{\Gamma} \\
\vec{u}
\end{array}\right) .
$$

Из леммы 3.1 получаем

СЛЕДСТВИЕ. Пространства $\mathbb{H}_{D} u \mathbb{V}_{2}^{1}\left(\Omega_{1}\right)$ совпадают, а нормы $\|\cdot\|_{D} u\|\cdot\|_{\mathbb{V}_{2}^{1}\left(\Omega_{1}\right)}$ в них әквивалентны.

Обозначим через $\mathbb{D}$ расширение по $\Phi$ ридрихсу оператора $\mathbb{D}_{0}$ (см. $\left.[15, \S 124]\right)$. Хотя область определния оператора $\mathbb{D}$ неизвестна, из определения следует, что область квадратного корня $\mathbb{D}^{1 / 2}$ совпадает с множеством функций

$$
U=\left(\begin{array}{c}
\vec{u}_{\Gamma} \\
\vec{u}
\end{array}\right), \quad \vec{u} \in \mathbb{V}_{2}^{1}\left(\Omega_{1}\right)=\mathbb{H}_{D}
$$

образуюших подпространство в пространстве $\mathbb{V}_{2}^{1 / 2}\left(S_{1}\right) \times \mathbb{V}_{2}^{1}\left(\Omega_{1}\right)$. Обратный оператор допускает матричное представление

$$
\mathbb{D}^{-1}=\left(\begin{array}{cc}
\Gamma \mathbb{B}_{0} & \Gamma \mathbb{B}_{1} \\
\mathbb{B}_{0} & \mathbb{B}_{1}
\end{array}\right)
$$

Здесь операторы $\mathbb{B}_{0}$ и $\mathbb{B}_{1}$ определены равенствами

$$
\mathbb{B}_{0} \vec{v}_{0}=\vec{u}_{0}, \quad \mathbb{B}_{1} \vec{v}_{1}=\vec{u}_{1}
$$

где функции $\vec{u}_{0}$ и $\vec{u}_{1}$ являются решениями задач

$$
\begin{array}{rlrlrl}
-\Delta^{*} \vec{u}_{0} & =0, & & -\Delta^{*} \vec{u}_{1} & =\vec{v}_{1}, \\
-\sigma_{n}\left(\vec{u}_{0}\right) & =\vec{v}_{0} & & \text { и } & -\sigma_{n}\left(\vec{u}_{1}\right) & =0 .
\end{array}
$$

В частности,

$$
\left(\mathbb{B}_{0} \vec{v}_{0}, \vec{w}_{0}\right)_{D}=\left(\vec{v}_{0}, \Gamma \vec{w}_{0}\right)_{\mathrm{E}_{2}\left(S_{1}\right)} \text { и }\left(\mathbb{B}_{1} \vec{v}_{1}, \vec{w}_{1}\right)_{D}=\left(\vec{v}_{1}, \vec{w}_{1}\right)_{\mathrm{E}_{2}\left(\Omega_{1}\right)} \cdot
$$

По аналогии с конструкцией оператора $\mathbb{A}$ в (2.5) рассматриваются обобщенные решения (принадлежащие $\mathbb{V}_{2}^{1}\left(\Omega_{1}\right)$ ) соответствующих задач для функций $\left\{\vec{v}_{0}, \vec{v}_{1}\right\} \in\left(L_{2}\left(S_{1}\right)\right)^{3} \times$ $\left(L_{2}\left(\Omega_{1}\right)\right)^{3}$. 
4. Редукция системы к уравнению в гильбертовом пространстве. Согласно определениям $(3.4),(3.5)$ равенства (3.2) и (3.3) можно переписать в виде

$$
-\vec{u}=\mu^{2} \mathbb{B}_{1} \frac{\partial^{2} \vec{u}}{\partial t^{2}}+\rho \mu^{2} \mathbb{B}_{0} \frac{\partial^{2}}{\partial t^{2}} \vec{N}\left(A_{10} \eta+A_{11} N \Gamma \vec{u}\right)+\mu^{2}(\rho-1) \mathbb{B}_{0} \vec{N}{ }_{3} \Gamma \vec{u} .
$$

Теперь вся система (0.1)-(0.5) запишется в виде

$$
\mathscr{T} w(t)+\mathscr{A} \ddot{w}(t)=0
$$

где $w(t) \in \mathscr{H}=L_{2}\left(S_{0}\right) \times \mathscr{H}_{D}$, и

$$
\begin{gathered}
\mathscr{A}=\mathscr{A}_{0}+\mathscr{A}_{1}, \quad \mathscr{A}_{0}:=\left(\begin{array}{cc}
A_{00} & A_{01} N \Gamma \\
\mathbb{B}_{0} \vec{N} A_{10} & \mathbb{B}_{0} \vec{N} A_{11} N \Gamma
\end{array}\right), \quad \mathscr{A}_{1}:=\frac{1}{\rho}\left(\begin{array}{ll}
0 & 0 \\
0 & \mathbb{B}_{1}
\end{array}\right), \\
\mathscr{T}:=\frac{1}{\rho \mu^{2}}\left(\begin{array}{cc}
\rho \mu^{2} I & 0 \\
0 & I+\mu^{2}(\rho-1) \mathbb{B}_{0} \vec{N}_{3} \Gamma
\end{array}\right), \quad w(t):=\left(\begin{array}{l}
\eta \\
\vec{u}
\end{array}\right) .
\end{gathered}
$$

Изучим свойства операторов $\mathscr{A}$ и $\mathscr{T}$.

Лемма 4.1. Oператор $\mathscr{A}$ положителен, а $\mathscr{T}$ равномерно положсителен в $\mathscr{H}$.

ДокАЗАТЕЛЬСТво. Непосредственно из определений получаем

$$
\begin{aligned}
\left(\mathscr{T}\left(\begin{array}{l}
\eta \\
\vec{u}
\end{array}\right),\left(\begin{array}{l}
\eta \\
\vec{u}
\end{array}\right)\right)_{\mathscr{H}} & =\|\eta\|_{L_{2}\left(S_{0}\right)}^{2}+\frac{1}{\rho \mu^{2}}\|\vec{u}\|_{D}^{2}+\frac{\rho-1}{\rho \mu^{2}}\left(n_{3} \Gamma \vec{u}_{3}, \Gamma \vec{u}_{3}\right)_{L_{2}\left(S_{1}\right)}^{2} \\
& \geqslant \min \left\{1, \frac{1}{\rho \mu^{2}}\right\}\left\|\left(\begin{array}{l}
\eta \\
\vec{u}
\end{array}\right)\right\|_{\mathscr{H}}^{2},
\end{aligned}
$$

так как $n_{3}<0$ и $\rho<1$. Далее,

$$
\begin{aligned}
\left(\mathscr{A}_{0}\left(\begin{array}{l}
\eta \\
\vec{u}
\end{array}\right),\left(\begin{array}{l}
\eta \\
\vec{u}
\end{array}\right)\right)_{\mathscr{H}}= & \left(A_{00} \eta, \eta\right)+\left(A_{01} N \Gamma \vec{u}, \eta\right) \\
& +\left(\mathbb{B}_{0} \vec{N} A_{10} \eta, \vec{u}\right)_{D}+\left(\mathbb{B}_{0} \vec{N} A_{11} N \Gamma \vec{u}, \vec{u}\right)_{D} \\
= & \left(A_{00} \eta, \eta\right)+\left(A_{01} u_{n}, \eta\right)+\left(A_{10} \eta, u_{n}\right)+\left(A_{11} u_{n}, u_{n}\right) \\
= & \left(\mathbb{A}\left(\begin{array}{c}
\eta \\
u_{n}
\end{array}\right),\left(\begin{array}{c}
\eta \\
u_{n}
\end{array}\right)\right)_{L_{2}(S)}>0, \\
\left(\mathscr{A}_{1}\left(\begin{array}{c}
\eta \\
\vec{u}
\end{array}\right),\left(\begin{array}{c}
\eta \\
\vec{u}
\end{array}\right)\right)_{\mathscr{H}}= & \frac{1}{\rho}\left(\mathbb{B}_{1} \vec{u}, \vec{u}\right)_{D}=\frac{1}{\rho}\|\vec{u}\|_{\mathrm{E}_{2}\left(\Omega_{1}\right)}^{2}>0 .
\end{aligned}
$$

Лемма доказана.

ЗАмЕчАниЕ 4.1. Если задачу рассматривать без учета сил гравитации (как это делалось в работе [6]), то слагаемое $\mu^{2}(\rho-1) u_{3} n_{3} \vec{e}_{3}$ в уравнении $(0.4)$ отсутствует. В этом случае построенньй оператор $\mathscr{T}$ ограничен и обратим (более того, $\left.\mathscr{T}=\operatorname{diag}\left(I, I /\left(\rho \mu^{2}\right)\right)\right)$, а уравнение (4.1) принимает вид

$$
w(t)+\mathscr{A}^{\prime} \ddot{w}(t)=0
$$

с положительным оператором $\mathscr{A}^{\prime}$. Для этого уравнения корректна задача Коши, решение которой выписьвается с помощью унитарной групп, генератором которой является расширение по Фридрихсу оператора $\left(\mathscr{A}^{\prime}\right)^{-1}$ (см., например, [16]). 
Пусть $w(t)$ - классическое решение уравнения (4.1). Под этим подразумеваем, что $w(t) \in W_{2}^{2}(\mathbb{R}, \mathscr{H}), w(t) \in \mathscr{D}(\mathscr{T}), \ddot{w}(t) \in \mathscr{D}(\mathscr{A})$. Тогда согласно теореме о промежуточных производных $\left[17\right.$, гл. 1] $\dot{w}(t) \in \mathscr{D}\left(\mathscr{A}^{1 / 2}\right)$. Следовательно, корректно определен функционал энергии решения $w(t)$

$$
E(t):=(\mathscr{A} \dot{w}(t), \dot{w}(t))_{\mathscr{H}}+(\mathscr{T} w(t), w(t))_{\mathscr{H}}
$$

Дифференцируя $E(t)$ и используя равенство $(4.1)$, получаем $\dot{E}(t)=0$, т.е. $E(t)=$ const. Более того, справедлив следующий результат.

ЛЕмма 4.2. Если $w(t)$ - классическое решение уравнения (4.1), то его энергия не зависит от $t$ и совпадает с функиионалом $T-\Pi$, определенным в $(0.9)$.

ДокАЗАТЕЛЬСТво. Вычислим функционал (4.2), воспользовавшись определениями операторов $\mathscr{A}$ и $\mathscr{T}$. После несложных преобразований получим утверждение леммы.

5. Спектр задачи. Естественно, что спектр задачи (0.1)-(0.6) следует определить как спектр пучка $\mathscr{L}(\lambda):=\mathscr{A}-\lambda \mathscr{T}$. Однако спектр этого пучка можно понимать в разных смыслах (см. [18]).

Например, классический спектр можно определить так. Возьмем в качестве области определения пучка $\mathscr{L}(\lambda)$ множество $\mathscr{D}(\mathscr{L})=\mathscr{D}\left(\mathscr{A}_{F}\right) \cap \mathscr{D}\left(T_{F}\right)$, где $\mathscr{A}_{F}$ и $\mathscr{T}_{F}-$ расширения по Фридрихсу операторов $\mathscr{A}$ и $\mathscr{T}$. Будем говорить, что $\lambda_{0} \in \mathbb{C}$ принадлежит классическому спектру $\sigma_{\mathrm{cl}}(\mathscr{L})$, если и только если оператор $\mathscr{A}-\lambda_{0} \mathscr{T}$, определенный на $\mathscr{D}(\mathscr{L})$, не имеет ограниченного обратного в $\mathscr{H}$. Обобщенный спектр $\sigma_{\mathrm{gen}}(\mathscr{L})$ определим как обычный спектр расширения по Фридрихсу оператора $\mathscr{A}=\mathscr{T}_{F}^{-1 / 2} \mathscr{A}_{F}^{1 / 2}$. Из определения следует, что $\sigma_{\text {gen }}(\mathscr{L}) \subset[0, \infty)$ и $\sigma_{\text {gen }}(\mathscr{L}) \supset \sigma_{\mathrm{cl}}(\mathscr{L})$.

Для поиска спектра задачи удобнее вернуться к пучку, определяемому системой (3.1)-(3.3). Заметим, что спектральная задача для пучка $\mathscr{L}(\lambda)=\mathscr{A}-\lambda \mathscr{T}$ в пространстве $\mathscr{H}$ эквивалентна системе (3.1)-(3.3) в пространстве $\mathscr{H}^{\prime}:=L_{2}\left(S_{0}\right) \times$ $\left(L_{2}\left(S_{1}\right)\right)^{3} \times\left(L_{2}\left(\Omega_{1}\right)\right)^{3}$ (если $-\partial^{2} / \partial t^{2}$ заменить на $\left.\lambda\right)$ и эквивалентность осуществляется оператором $I \oplus \mathbb{D}$ (это следует из определения оператора $\mathbb{D}$ ). Поэтому достаточно изучить спектр линейного пучка

$$
\mathscr{L}^{\prime}(\lambda)=\mathscr{A}^{\prime}-\lambda \mathscr{T}^{\prime}
$$

в пространстве $\mathscr{H}^{\prime}$ с операторами

$$
\mathscr{A}^{\prime}:=\left(\begin{array}{ccc}
I & 0 & 0 \\
0 & -\mu^{2}(\rho-1) N_{3} & \sigma_{n} \\
0 & 0 & \Delta^{*}
\end{array}\right), \quad \mathscr{T}^{\prime}:=\left(\begin{array}{ccc}
A_{00} & A_{01} N & 0 \\
-\rho \mu^{2} \vec{N} A_{10} & -\rho \mu^{2} \vec{N} A_{11} N & 0 \\
0 & 0 & -\mu^{2}
\end{array}\right)
$$

Сначала рассмотрим случай плоского дна. В этом случае проблем с областями определения операторов не возникает (не нужно делать акцент на фридрихсовских расширениях, в отличие от случая, когда поверхность $S_{1}$ имеет изломы). Пусть $H\left(x_{1}, x_{2}\right) \equiv h>0$. Естественно применить к пучку $\mathscr{L}^{\prime}(\lambda)$ преобразование $Ф$ урье $\mathscr{F}$ по отношению к переменным $x_{1}$ и $x_{2}$ :

$$
(\mathscr{F} f)\left(p_{1}, p_{2}\right)=\frac{1}{2 \pi} \int_{\mathbb{R}^{2}} e^{-i\left(p_{1} x_{1}+p_{2} x_{2}\right)} f\left(x_{1}, x_{2}\right) d x_{1} d x_{2}
$$


Прямые вычисления приводят к следующему результату:

$$
\begin{aligned}
\mathscr{F} \mathbb{A} \mathscr{F}^{-1} & =(|p| \operatorname{sh}(|p| h))^{-1}\left(\begin{array}{cc}
\operatorname{ch}(|p| h) & 1 \\
1 & \operatorname{ch}(|p| h)
\end{array}\right), \\
\mathscr{F} \Delta^{*} \mathscr{F}^{-1} & =N_{0} \frac{\partial^{2}}{\partial x_{3}^{2}}+i N_{1} \frac{\partial}{\partial x_{3}}-N_{2}=: L\left(\frac{\partial}{\partial x_{3}}\right) \\
\mathscr{F} \sigma_{n} \mathscr{F}^{-1} & =-N_{0} \frac{\partial}{\partial x_{3}}-i N_{1}^{\prime}=: M\left(\frac{\partial}{\partial x_{3}}\right)
\end{aligned}
$$

где

$$
\begin{gathered}
N_{0}=\left(\begin{array}{ccc}
1 & 0 & 0 \\
0 & 1 & 0 \\
0 & 0 & c^{2}
\end{array}\right), \quad N_{1}=\left(c^{2}-1\right)\left(\begin{array}{ccc}
0 & 0 & p_{1} \\
0 & 0 & p_{2} \\
p_{1} & p_{2} & 0
\end{array}\right), \\
N_{1}^{\prime}=\left(\begin{array}{ccc}
0 & 0 & p_{1} \\
0 & 0 & p_{2} \\
\left(c^{2}-2\right) p_{1} & \left(c^{2}-2\right) p_{2} & 0
\end{array}\right), \quad N_{2}=\left(c^{2}-1\right)\left(\begin{array}{ccc}
p_{1}^{2} & p_{1} p_{2} & 0 \\
p_{1} p_{2} & p_{2}^{2} & 0 \\
0 & 0 & 0
\end{array}\right)+|p|^{2} I .
\end{gathered}
$$

Если $\left\{\eta\left(x_{1}, x_{2}\right), \vec{u}(x)\right\}$ - собственные функции пучка $\mathscr{L}(\lambda)$ или решения системы (3.1)-(3.3) вида

$$
\vec{u}(x, t)=\vec{u}(x) e^{i \omega t}, \quad \eta\left(x_{1}, x_{2}, t\right)=\eta\left(x_{1}, x_{2}\right) e^{i \omega t}, \quad \omega=\lambda^{1 / 2},
$$

то после преобразования Фурье по переменным $p_{1}, p_{2}$ для образов Фурье

$$
\widehat{u}\left(p_{1}, p_{2}, z\right):=\mathscr{F} \vec{u}\left(x_{1}, x_{2}, x_{3}+h\right), \quad \widehat{\eta}\left(p_{1}, p_{2}\right):=\mathscr{F} \eta\left(x_{1}, x_{2}\right)
$$

получаем равенства

$$
\begin{aligned}
& \widehat{\eta}-\frac{\omega^{2}}{|p| \operatorname{sh}(|p| h)}\left(\operatorname{ch}(|p| h) \widehat{\eta}-\widehat{u}_{3}\left(p_{1}, p_{2}, 0\right)\right)=0 \\
& \vec{N}\left\{\mu^{2}(\rho-1) \widehat{u}_{3}\left(p_{1}, p_{2}, 0\right)-\frac{\rho \mu^{2} \omega^{2}}{|p| \operatorname{sh}(|p| h)}\left(\widehat{\eta}-\operatorname{ch}(|p| h) \widehat{u}_{3}\left(p_{1}, p_{2}, 0\right)\right)\right\} \\
& \quad=\left.M\left(\frac{\partial}{\partial z}\right) \widehat{u}\left(p_{1}, p_{2}, z\right)\right|_{z=0} \\
& -\omega^{2} \mu^{2} \widehat{u}\left(p_{1}, p_{2}, z\right)=L\left(\frac{\partial}{\partial z}\right) \widehat{u}\left(p_{1}, p_{2}, z\right) .
\end{aligned}
$$

Для фиксированного $p \in \mathbb{R}^{2}$ систему (5.4)-(5.6) можно рассматривать как задачу на собственные значения для линейного пучка

$$
\widehat{\mathscr{A}}_{p}-\lambda \widehat{\mathscr{T}}_{p}=: \widehat{\mathscr{L}}_{p}(\lambda)
$$

в пространстве $\mathscr{H}^{\prime \prime}:=\mathbb{C} \times \mathbb{C} \times L_{2}(-\infty, 0]$. Другими словами, мы должны разложить пространство $\mathscr{H}^{\prime}$ и линейный пучок $\widehat{\mathscr{L}}(\lambda):=\mathscr{F}\left(\mathscr{A}^{\prime}-\lambda \mathscr{T}^{\prime}\right) \mathscr{F}^{-1}$ в прямой интеграл (см. $[19$, гл. ХІІІ])

$$
\mathscr{H}^{\prime}=\int_{\mathbb{R}^{2}}^{\oplus} \mathscr{H}^{\prime \prime} d p, \quad \widehat{\mathscr{L}}(\lambda)=\int_{\mathbb{R}^{2}}^{\oplus} \widehat{\mathscr{L}}_{p}(\lambda) d p
$$

где $d p$ - мера Лебега в $\mathbb{R}^{2}$. Из теории прямых разложений операторов следует, что $\widehat{\mathscr{L}}^{-1}\left(\lambda_{0}\right)$ существует и ограничен в $\mathscr{H}^{\prime}$, если и только если $\widehat{\mathscr{L}}_{p}^{-1}\left(\lambda_{0}\right)$ ограничен почти для всех $p \in \mathbb{R}^{2}$ и $\int_{\mathbb{R}^{2}}\left\|\widehat{\mathscr{L}}_{p}^{-1}\left(\lambda_{0}\right)\right\| d p<\infty$. В частности, справедлив следующий результат (cp. [19, теорема XIII.85]). 
ЛЕмма 5.1. Пусть точка $\lambda_{0}$ такая, что для любого $\varepsilon>0$ множество

$$
\left\{p \in \mathbb{R}^{2} \mid \sigma_{\mathrm{cl}}\left(\widehat{\mathscr{L}}_{p}(\lambda)\right) \cap\left\{\lambda|| \lambda-\lambda_{0} \mid \leqslant \varepsilon\right\}\right\}
$$

имеет полохительную меру Лебега. Тогда $\lambda_{0} \in \sigma_{\mathrm{cl}}(\widehat{\mathscr{L}})$.

Таким образом, чтобы доказать равенство $\sigma_{\mathrm{cl}}(\widehat{\mathscr{L}})=[0, \infty)$ достаточно показать, что при любом $\lambda_{0} \in[0, \infty)$ вьполняются условия леммы 5.1. Для этого изучим спектр линейного пучка $\hat{\mathscr{L}}_{p}(\lambda)$. Очевидно, функция $y\left(p_{1}, p_{2}\right) e^{i k_{0} z}$ является решением уравнения $(5.6)$, если и только если $k_{0}$ и $y\left(p_{1}, p_{2}\right)$ являются собственным значением и собственной функцией квадратичного матричного пучка операторов в пространстве $\mathbb{C}^{3}$ :

$$
\mathscr{L}(k, \omega):=k^{2} N_{0}+k N_{1}+N_{2}-\omega^{2} \mu^{2} I
$$

Вычислив детерминант матрицы $\mathscr{L}(k, \omega)$, получим следующие собственные пары (ветвь корня фиксируется условием $\arg \sqrt{z} \in[0, \pi)$ при $z \neq 0)$ :

$$
\begin{gathered}
k=k_{1}:=\sqrt{\frac{\omega^{2} \mu^{2}}{c^{2}}-|p|^{2}}, \quad y_{1}^{+}=\left(p_{1}, p_{2}, k_{1}\right) ; \quad k=-k_{1}, \quad y_{1}^{-}=\left(p_{1}, p_{2},-k_{1}\right) \\
k=k_{2}:=\sqrt{\omega^{2} \mu^{2}-|p|^{2}}, \quad y_{2}^{+}=\left(p_{2},-p_{1}, 0\right) ; \quad k=-k_{2}, \quad y_{2}^{-}=\left(p_{2},-p_{1}, 0\right) \\
k=k_{2}, \quad y_{3}^{+}=\left(p_{1} k_{2}, p_{2} k_{2},-|p|^{2}\right) ; \quad k=-k_{2}, \quad y_{3}^{-}=\left(p_{1} k_{2}, p_{2} k_{2},|p|^{2}\right) .
\end{gathered}
$$

Поэтому общее решение уравнения (5.6) имеет вид

$$
\begin{aligned}
\widehat{u}\left(p_{1}, p_{2}, z\right)= & A_{1}^{+} y_{1}^{+} e^{i k_{1} z}+A_{1}^{-} y_{1}^{-} e^{-i k_{1} z}+A_{2}^{+} y_{2}^{+} e^{i k_{2} z}+A_{2}^{-} y_{2}^{-} e^{-i k_{2} z} \\
& +A_{3}^{+} y_{3}^{+} e^{i k_{2} z}+A_{3}^{-} y_{3}^{-} e^{-i k_{2} z}
\end{aligned}
$$

Из уравнения (5.5) следует, что вектор $\left.M(\partial / \partial z) \widehat{u}\left(p_{1}, p_{2}, z\right)\right|_{z=0}$ пропорционален вектору $\vec{N}$, что приводит к следуюшим соотношениям для коэффициентов в $(5.8)$ :

$$
\begin{gathered}
A_{2}^{+}=A_{2}^{-} \\
2 k_{1}\left(A_{1}^{+}-A_{1}^{-}\right)=\left(|p|^{2}-k_{2}^{2}\right)\left(A_{3}^{+}-A_{3}^{-}\right) .
\end{gathered}
$$

Из уравнения (5.4) выразим вектор $\widehat{\eta}$

$$
\widehat{\eta}=-\frac{\omega^{2}}{|p| \operatorname{sh}(|p| h)-\omega^{2} \operatorname{ch}(|p| h)} \widehat{u}_{3}\left(p_{1}, p_{2}, 0\right)
$$

и внесем его в (5.5):

$$
\begin{array}{r}
i\left(c^{2}-2\right)\left(p_{1} \widehat{u}_{1}\left(p_{1}, p_{2}, 0\right)+p_{2} \widehat{u}_{2}\left(p_{1}, p_{2}, 0\right)\right)+\left.c^{2} \frac{\partial \widehat{u}_{3}\left(p_{1}, p_{2}, z\right)}{\partial z}\right|_{z=0} \\
=\left\{\mu^{2}(\rho-1)-\frac{\rho \mu^{2} \omega^{2}}{|p|} \frac{|p| \operatorname{ch}(|p| h)-\omega^{2} \operatorname{sh}(|p| h)}{|p| \operatorname{sh}(|p| h)-\omega^{2} \operatorname{ch}(|p| h)}\right\} \widehat{u}_{3}\left(p_{1}, p_{2}, 0\right) .
\end{array}
$$


Подставив (5.8) в последнее уравнение, мы получим третье соотношение (дополнительное к (5.9) и (5.10)) для коэффициентов $A_{j}^{ \pm}, j=1,2,3$ :

$$
\begin{aligned}
& i\left(\omega^{2} \mu^{2}-2|p|^{2}\right)\left(A_{1}^{+}-A_{1}^{-}\right)-2 i|p|^{2} k_{2}\left(A_{3}^{+}+A_{3}^{-}\right) \\
& \quad=\left\{\mu^{2}(\rho-1)-\frac{\rho \mu^{2} \omega^{2}}{|p|} \frac{|p| \operatorname{ch}(|p| h)-\omega^{2} \operatorname{sh}(|p| h)}{|p| \operatorname{sh}(|p| h)-\omega^{2} \operatorname{ch}(|p| h)}\right\}\left(k_{1}\left(A_{1}^{+}-A_{1}^{-}\right)-|p|^{2}\left(A_{3}^{+}-A_{3}^{-}\right)\right) .
\end{aligned}
$$

Теперь мы можем найти все ограниченные по $x$ решения системы $(5.4)-(5.6)$ для фиксированного $|p|$. Рассмотрим следующие случаи:
1) $\omega^{2}<\frac{|p|^{2}}{\mu^{2}}$
2) $\frac{|p|^{2}}{\mu^{2}} \leqslant \omega^{2}<\frac{c^{2}|p|^{2}}{\mu^{2}}$
3) $\omega^{2}>\frac{c^{2}|p|^{2}}{\mu^{2}}$.

В первом случае $k_{j}=i \varkappa_{j}$, где $\varkappa_{j}>0, j=1,2$. Поэтому ограниченное при $x_{3} \rightarrow-\infty$ решение возможно лишь если $A_{1}^{+}=A_{2}^{+}=A_{3}^{+}=0$. Учитьвая этот факт, а также соотношения (5.9), (5.10) и (5.12), получаем следующее "дисперсионное соотношение" для собственньх частот (cp. [11]):

$$
4 i|p|^{2} k_{1} k_{2}+i\left(\omega^{2} \mu^{2}-2|p|^{2}\right)^{2}+\omega^{2} \mu^{4}(\rho-1) k_{1}=\frac{\rho \mu^{4} \omega^{4} k_{1}}{|p|} \frac{|p| \operatorname{ch}(|p| h)-\omega^{2} \operatorname{sh}(|p| h)}{|p| \operatorname{sh}(|p| h)-\omega^{2} \operatorname{ch}(|p| h)}
$$

Любое решение $\omega_{0}<|p|^{2} / \mu^{2}$ этого уравнения порождает собственное значение $\lambda=\omega_{0}^{2}$ пучка $\widehat{\mathscr{L}}_{p}^{\prime}(\lambda)$, которому соответствует экспоненциально убьвающий по $z$ вектор

$$
\frac{|p|^{2}-k_{2}^{2}}{2 k_{1}}\left(\begin{array}{c}
p_{1} \\
p_{2} \\
-k_{1}
\end{array}\right) e^{-i k_{1} z}+\left(\begin{array}{c}
p_{1} k_{2} \\
p_{2} k_{2} \\
|p|^{2}
\end{array}\right) e^{-i k_{2} z}
$$

где $k_{1}$ и $k_{2}$ определены соотношением (5.7). Известно (ср. [11]), что уравнение (5.13) имеет два решения в интервале $\left(0,|p|^{2} / \mu^{2}\right)$, т.е. спектр пучка $\widehat{\mathscr{L}}_{p}^{\prime}(\lambda)$ в этом интервале состоит из двух собственных значений.

Во втором случае $k_{2}>0$ и мы получаем решения с $A_{2}^{+}=A_{2}^{-}$вида

$$
y_{1}\left(p_{1}, p_{2}, z\right)=A_{2}^{ \pm}\left(\begin{array}{c}
p_{2} \\
-p_{1} \\
0
\end{array}\right) \sin k_{2} z
$$

Одномерное пространство ограниченных решений, линейно независимых с $y_{1}$, получается подстановкой $A_{1}^{+}=A_{2}^{+}=A_{2}^{-}=0$. Соответствуюшие решения имеют вид

$$
y_{2}\left(p_{1}, p_{2}, z\right)=A_{1}^{-}\left(\begin{array}{c}
p_{1} \\
p_{2} \\
-k_{1}
\end{array}\right) e^{-i k_{1} z}+A_{3}^{+}\left(\begin{array}{c}
p_{1} k_{2} \\
p_{2} k_{2} \\
-|p|^{2}
\end{array}\right) e^{i k_{2} z}+A_{3}^{-}\left(\begin{array}{c}
p_{1} k_{2} \\
p_{2} k_{2} \\
|p|^{2}
\end{array}\right) e^{-i k_{2} z}
$$


с коэффициентами $A_{1}^{-}, A_{3}^{+}, A_{3}^{-}$, удовлетворяющими уравнениям (5.10) и (5.12). Теперь, фиксируя число $\lambda_{0}=\omega_{0}^{2} \in\left[|p|^{2} / \mu^{2}, c^{2}|p|^{2} / \mu^{2}\right)$, с помощью стандартной процедуры срезки можно построить последовательность Вейля для оператора $\widehat{\mathscr{L}}_{p}\left(\lambda_{0}\right)$, т.е. последовательность векторов $w_{n}:=\left(\widehat{\eta}_{n}(p), \widehat{u}_{3, n}(p, 0), \widehat{u}_{n}(p, z)\right)$, удовлетворяющих условиям

$$
\left\|w_{n}\right\|_{\mathscr{H}^{\prime \prime}} \geqslant 1, \quad\left\|\widehat{\mathscr{L}}_{p}\left(\lambda_{0}\right) w_{n}\right\| \rightarrow 0 \text { при } n \rightarrow \infty .
$$

Это означает, что любое число $\lambda_{0} \in\left[|p|^{2} / \mu^{2}, c^{2}|p|^{2} / \mu^{2}\right)$ принадлежит спектру (причем существенному спектру) пучка $\widehat{\mathscr{L}}_{p}(\lambda)$.

Наконец, при $\omega^{2}>c^{2}\left|p^{2}\right| / \mu^{2}$ в дополнение к функциям $Y_{1}\left(p_{1}, p_{2}, z\right)$ и $Y_{2}\left(p_{1}, p_{2}, z\right)$ мы имеем линейно независимое решение вида

$$
y_{3}\left(p_{1}, p_{2}, z\right)=A_{1}^{+}\left(\begin{array}{c}
p_{1} \\
p_{2} \\
k_{1}
\end{array}\right) e^{-i k_{1} z}+A_{3}^{+}\left(\begin{array}{c}
p_{1} k_{2} \\
p_{2} k_{2} \\
-|p|^{2}
\end{array}\right) e^{i k_{2} z}+A_{3}^{-}\left(\begin{array}{c}
p_{1} k_{2} \\
p_{2} k_{2} \\
|p|^{2}
\end{array}\right) e^{-i k_{2} z}
$$

где коэффициенты $A_{1}^{+}, A_{3}^{+}, A_{3}^{-}$удовлетворяют соотношениям (5.10) и (5.12) при $A_{1}^{-}=0$. Как и ранее, с помощью процедуры срезки получаем, что любое число $\lambda_{0} \in\left[c^{2}|p|^{2} / \mu^{2}, \infty\right)$ есть точка существенного спектра пучка $\widehat{\mathscr{L}}_{p}(\lambda)$.

Проведенные вькладки приводят нас к следующим результатам.

ТЕОРема 5.1. При любом фиксированном $p=\left(p_{1}, p_{2}\right) \in \mathbb{R}^{2}$ классический спектр пучка $\widehat{\mathscr{L}}_{p}(\lambda)$ следующии:

а) два собственных значения $\omega_{ \pm}^{2} \in\left(0,|p|^{2} / \mu^{2}\right)$, являющиеся решениями дисперсионного уравнения, причем отвечающие им собственные функиии определень посредством (5.14) и (5.11);

b) существенный спектр кратности 2 , покрывающий сегмент $\left[|p|^{2} / \mu^{2}, c^{2}|p|^{2} / \mu^{2}\right)$;

c) существенный спектр кратности 3 , покрывающий интервал $\left[c^{2}|p|^{2} / \mu^{2}, \infty\right)$.

Теорема 5.2. Пусть дно $S_{1}$ есть плоскость, ортогональная направлению $x_{3}$. Тогда спектр пучка $\mathscr{L}(\lambda)$ (а значит, и спектр задачи (0.1)-(0.6)) совпадает со всей полохительной осъю.

ДокАЗАТЕЛЬСТВо. Это утверждение есть следствие леммы 5.1 и теоремы 5.1.

Рассмотрим теперь случай, когда поверхность дна отличается от плоской лишь на компакте (т.е. $H\left(x_{1}, x_{2}\right)=$ const при $|x|$ достаточно больших). В этом случае при любом фиксированном $\lambda_{0} \in \mathbb{C}$ и $p \in \mathbb{R}^{2}$ оператор $\widehat{\mathscr{L}}_{p}\left(\lambda_{0}\right)$ является компактным возмущением соответствующего оператора для случая плоского дна. Поэтому существенные спектры линейных пучков $\widehat{\mathscr{L}}_{p}(\lambda)$ для плоского и компактно возмущенного дна совпадают и при $p \rightarrow 0$ стремятся к $[0, \infty)$. Кроме того, спектр вещественный и отрищательных собственных значений быть не может. Поэтому справедлив следующий результат.

Теорема 5.3. В случае компактно возмущенного плоского дна спектр пучка $\mathscr{L}(\lambda)$ (или спектр задачи (0.1)-(0.6)) совпадает со всей положительной полуосью $[0, \infty)$. 


\section{СПИСОК ЦИТИРОВАННОЙ ЛИТЕРАТУРЫ}

[1] Подъяпольский Г. С. Возбуждение длинной гравитационной волны в океане сейсмическим источником в коре // Изв. АН СССР. Физика Земли. 1968. № 1. С. 7-24.

[2] Зволинский Н. В. О сейсмическом механизме возбуждения волн цунами // Изв. АН СССР. Физика Земли. 1986. № 3. С. 3-15.

[3] Зволинский М. В., Никитин И. С., Секерж-Зенькович С. Я. Генерация волн цунами и волн Релея гармоническим центром расширения // Изв. АН СССР. Физика Земли. 1991. № 2. C. 34-44.

[4] Асланян А. Г., Васильев Д. Г., Лидский В. Б. Частота свободных колебаний тонкой оболочки, взаимодействующей с жидкостью // Функцион. анализ и его прилож. 1981. Т. 15. № 3. С. 1-9.

[5] Молотков И.А., Крауклисс П. В. Смешанные поверхностные волны на границе упругой среды и жидкости // Изв. АН СССР. Физика Земли. 1971. № 8. С. 3-11.

[6] Фрагела А.К. К задаче о движении идеальной жидкости в неограниченном упругом бассейне // Дифференц. уравнения. 1989. Т. 25. № 8. С. 1417-1426.

[7] Уизем Дж. Линейные и нелинейные волны. М.: Мир, 1977.

[8] Бердичевский Б. Л. Вариационные принципы механики сплошной среды. М.: Наука, 1983.

[9] Захаров В. Е. Устойчивость периодических волн конечной амплитуды на поверхности глубокой жидкости // ПМТФ. 1968. №2. С. 86-94.

[10] Luke J. C. A variational principle for a fluid with a free surface // J. Fluid Mech. 1967. V. 27. P. 395-397.

[11] Доброхотов С. Ю., Толстова О. Л., Чудинович И. Ю. Волны в жидкости на упругом основании. Теория существования и точные решения // Матем. заметки. 1993. Т. 54. № 6 . C. $33-55$.

[12] Мазья В. Г. Пространства Соболева. Л.: Изд-во Ленингр. ун-та, 1985.

[13] Стейн И. Сингулярные интегралы и дифференциальные свойства функций. М.: Мир, 1973.

[14] Кондратьев В.А., Олейник О.А. Краевые задачи для системы теории упругости в неограниченных областях. Неравенства Корна // УМН. 1988. Т. 43. № 5. С. 55-98.

[15] Рисс Ф., Секефальви-Надь Б. Лекции по функциональному анализу. М.: Мир, 1979.

[16] Крейн С. Г. Линейные дифференциальные уравнения в банаховом пространстве. М.: Наука, 1967.

[17] Лионс ЖК. Л., Мадженес Э. Неоднородные граничные задачи и их приложения. М.: Мир, 1971.

[18] Shkalikov A. A. Operator pencils arising in elasticity and hydrodynamics. Instability index formula // Operator Theory: Advances and Applications. V. 87. Basel: Birkhäuser Verlag, 1996. P. 258-285.

[19] Рид М., Саймон Б. Методы современной математической физики. Т. 4. М.: Мир, 1982.

(Р. О. Гринив) Институт прикладных проблем механики и математики

Поступило

НАН Украины, г. Львов

07.12.1999

(С. Ю. Доброхотов) Институт проблем механики РАН

(А. А. Шкаликов) Московский государственный университет

им. М. В. Ломоносова 